\title{
Response times and eye movements in feature and conjunction search as a function of target eccentricity
}

\author{
CHARLES T. SCIALFA and KENNETH M. JOFFE \\ University of Calgary, Calgary, Alberta, Canada
}

\begin{abstract}
In four experiments, saccadic eye movements, reaction times (RTs), and accuracy were measured as observers searched for feature or conjunction targets presented at several eccentricities. A conjunction search deficit, evidenced by a large eccentricity effect on RTs, accuracy, and number of saccades, was seen in Experiments $1 \mathrm{~A}$ and 1B. Experiment 2 indicated that, when saccades were precluded, there was an even larger eccentricity effect for conjunction search targets. In Experiment 3 , practice in a conjunction search task allowed both RT and number of saccades to become independent of eccentricity. Additionally, there was evidence of feature-based selectivity in that observers were more likely to fixate distractors that had the same contrast as the target. Results are consistent with the view that the oculomotor and attentional systems are functionally linked and provide constraints for models of visual attention and search.
\end{abstract}

Theories of visual attention and search often emphasize the relation between reaction time (RT) and the number of items in a display. For example, Treisman and her colleagues (Treisman, 1988; Treisman \& Gormican, 1988; Treisman \& Sato, 1990; Treisman \& Souther, 1985) have developed and extended feature integration theory (FIT), relying largely on the identification of those search conditions that produce negligible or substantial display size effects on RT. Alternative models of attention and search (see, e.g., Cave \& Wolfe, 1989; Duncan \& Humphreys, 1989; Wolfe, 1994) similarly rely on assessments of display size effects. Additionally, models of automaticity (see, e.g., Schneider, Pimm-Smith, \& Worden, 1994; Schneider \& Shiffrin, 1977) have used display size effects on RT to follow observers as they develop an automatic attentional response.

While display size effects provide valuable information about the processes underlying search, it has been suggested recently that target location also plays a significant role in target acquisition. Eccentricity effects have been reported in difficult feature search (Scialfa, Thomas, \& Joffe, 1994; Viviani \& Swensson, 1982), in which targets are defined by a feature value on one dimension. Larger eccentricity effects have been observed in orientation $X$ color conjunction search (Carrasco, Evert, Chang, \& Katz, $1995)$ and orientation $\times$ contrast polarity (i.e., white vs. black) conjunction search (Scialfa \& Joffe, 1997), in which the target is defined by its multiple feature values, each typically shared by half of the distractors.

This research was supported by a grant from the Natural Science and Engineering Research Council of Canada. K.M.J. is now at the School of Medicine, University of Alberta. Correspondence should be addressed to C. T. (Chip) Scialfa, Department of Psychology, University of Calgary, Calgary, AB, T2N 1N4 Canada (e-mail; scialfa@acs.ucalgary.ca).
In the experiments described here, we examined search performance when the target was presented at display eccentricities ranging from $3.82^{\circ}$ to $13.94^{\circ}$. Unlike Carrasco et al. (1995) and Viviani and Swensson (1982), we measured both feature and conjunction search in the same individuals. In addition, several dependent variables-manual RT, response accuracy, number of saccades, and fixation duration - were determined. In carrying out these studies, we addressed several questions about the oculomotor and attentional processes underlying visual search. First, what is the relation between target eccentricity and the dependent measures typically gathered in search experiments (i.e., response accuracy and RT)? Second, does oculomotor involvement change similarly, as would be expected if the attentional and oculomotor systems were linked (Henderson, 1993; Henderson, Pollatsek, \& Rayner, 1989; Hoffman, in press; Morrison, 1984)? Finally, does experience with conjunction search produce a change in the oculomotor and attentional demands of the task?

\section{Visual Search and Target Eccentricity}

A considerable body of evidence indicates that search performance depends on target location in a conditionspecific way. Scialfa, Kline, and Lyman (1987) asked observers to discriminate a $\mathrm{T}$ and an $\mathrm{O}$ embedded in 0,2 , or $17 \mathrm{Xs}$. Targets were located between $0^{\circ}$ and $10.5^{\circ}$ from fixation, and, to prevent eye movements, displays were presented for $125 \mathrm{msec}$. Both RT and errors increased with target eccentricity, but only when the target was embedded in a large number of distractors. The eccentricity effect is reflected in localization errors as well (Scialfa \& Kline, 1988). Similarly, Carrasco et al. (1995) observed that under both unlimited viewing (Experiment 1 ) and brief viewing (Experiments 2 and 3), color $\times$ orientation con- 
junction search produced large eccentricity effects on RT and accuracy.

A few investigations have examined the eye movements made during search for eccentric targets. Viviani and Swensson (1982) measured saccadic accuracy and latency when observers searched for a "star" presented at $4.4^{\circ}-13.2^{\circ}$ from fixation in circles of equal area. Performance was independent of target location until eccentricity exceeded $11^{\circ}$, after which there was a pronounced increase in saccadic latency and the probability of an error in the direction of the initial saccade. Scialfa et al. (1994) reported that the number of saccades made prior to a correct response increased with eccentricity when an oriented line was embedded in heterogeneous distractors. More recently, Scialfa and Joffe (1997) examined both feature and conjunction search (contrast polarity $\times$ orientation) as a function of target eccentricity. Among the young adult observers who were tested, RTs and saccades were independent of eccentricity in both feature search conditions. In contrast, conjunction search produced large eccentricity effects on both measures.

These eccentricity effects can be incorporated by models in which search is conducted with an attentional spotlight (Juola, Bouwhuis, Cooper, \& Warner, 1991; Posner, 1980 ) or zoom lens (Eriksen \& St. James, 1986), whereby the locus of attention must change to process objects falling outside the spotlight. Feature search is thought to arise when attention is distributed widely: RTs and the number of saccades should be independent of eccentricity (Binello, Mannan, \& Ruddock, 1995; Scialfa \& Joffe, 1997; Scialfa et al., 1994). In contrast, conjunction search often requires that attention be focused narrowly, perhaps on individual items. When attention is finely focused, both RT and number of saccades should increase with target eccentricity. These expectations are based on two assumptions: (1) The attentional spotlight can be described as a gradient, and (2) the oculomotor and attentional systems are closely coupled. Both of these assumptions warrant discussion.

Processing efficiency may not be constant within the attentional spotlight, but appears to decline with distance from the focus of attention in a gradient-like manner (Downing \& Pinker, 1985; Egly \& Homa, 1984; Eriksen \& Schultz, 1979; Rizzolatti, Riggio, Dascola, \& Umiltà, 1987; but see Kim \& Cave, 1995). Because observers are expected to have a narrow focus of attention in conjunction search, at display onset, elements occupying more eccentric locations will fall on a less sensitive area within, or completely outside of the attentional spotlight. Furthermore, it can be shown that regardless of where attention is focused, elements occupying more eccentric positions within the display will, on average, be more distant from that focus and thus will have reduced salience. Reduced salience or attention attraction strength (Schneider, 1985) implies that eccentric targets are unlikely to influence subsequent shifts of attention. As such, identification of them will take longer and more eye movements will be made.

\section{Functional Relations Between Attentional and Oculomotor Mechanisms}

We have asserted that eye movements will be more numerous for conjunction search targets presented away from the center of the display. The location of the attentional spotlight may shift in the absence of eye movements (Goldberg \& Bruce, 1985; Posner, 1980; Posner, Snyder, \& Davidson, 1980; Sperling \& Reeves, 1986), but there is ample evidence that the attentional and oculomotor systems are functionally related. It is difficult to produce an eye movement that directionally opposes a shift of attention (Braun \& Breitmeyer, 1988; Deubel \& Schneider, 1996; Hoffman \& Subramaniam, 1995; Khurana \& Kowler, 1987; Kowler, Anderson, Dosher, \& Blaser, 1995; Sheppard, Findlay, \& Hockey, 1986) or is in the direction corresponding to an attentional deficit (Pierrot-Diseilligny, Rivaud, Penet, \& Rigolet, 1987). In addition, both moving window (Henderson et al., 1989) and reading span studies (Henderson \& Ferreira, 1990; McConkie \& Rayner, 1975) provide evidence that extrafoveal acquisition of information is largely restricted to that location to which the eyes are about to move.

Attentional involvement in saccade generation has been documented at the physiological level as well. Oculomotor cells in the superior colliculus, frontal eye fields, and parietal lobe show enhanced activity to stimuli that must be attended (Goldberg \& Bruce, 1985; Goldberg \& Wurtz, 1972; Lynch, Mountcastle, Talbot, \& Yin, 1977). Furthermore, the ease of obtaining an electrically elicited saccade depends on the correspondence between the movement vector of the oculomotor cell and the location to which attention has been directed (Goldberg, Bushnell, \& Bruce, 1986; Lynch et al., 1977; Munoz \& Wurtz, 1992; Schiller \& Sandell, 1983; Shibutani, Sakata, \& Hyvarinen, 1984; Sparks \& Mays, 1983).

Of greatest relevance to the present discussion, additional support for an attentional-oculomotor link comes from the search literature. Fixation durations and saccade number increase, while saccade amplitudes decrease with greater intradisplay complexity (Jacobs, 1986; Locher \& Nodine, 1978; Rayner \& Fisher, 1987). Eye movements are concentrated at those display locations containing the most task-relevant information (Latimer, 1990; Saitoh \& Okazaki, 1990) and are influenced by target location expectancies (see R. M. Steinman, 1986). When attention is narrowly distributed, it is difficult to generate an eye movement to an eccentric target, and when saccades are made, their endpoints are less influenced by nontarget objects (Findlay \& Kapoula, 1992; Miura, 1990). Finally, saccades are executed to detect an eccentric feature search target embedded in heterogeneous distractors (Scialfa et al., 1994) and to identify a conjunction search target (Scialfa \& Joffe, 1997).

Taken as a whole, these studies lend credence to Henderson's (1993; Henderson et al., 1989) sequential attention model, which postulates a strong coupling between the attentional and oculomotor systems. Specifically, at the beginning of each new fixation, attentional resources 
are concentrated near fixation. After some criterion level of processing has been reached, attention is disengaged from the current fixation and moved to some other display area on the basis of a weighting of salient features. This new location is used to program the coordinates of the next saccade. An important question is whether the eye movements made are necessary for efficient search performance in difficult search conditions.

Other data can be brought to bear on the issue of oculomotor-attentional dependencies. If there is no coupling of the attentional and eye movement systems, there is little reason to expect a relation between the number of saccades an observer makes and the duration of the fixations separating saccades. Alternatively, one might well imagine that when many saccades are made, little information is processed per fixation and that when few saccades are made, the processing demands per fixation are similarly increased. Under this view, an inverse relation between saccade (fixation) number and fixation durations would be expected. Nattkemper and Prinz (1986) reported this result in a continuous letter search task and Scialfa and Joffe (1997) found that power functions with negative exponents described the relation between number of saccades and fixation duration in both feature and conjunction search. They also found a greater $y$-intercept in conjunction search but the exponent was not related to search conditions; at a constant number of eye movements, fixation duration was longer in the most demanding search condition. In the present experiments, we examined this relation again, carrying out a more thorough analysis of the fixation data.

\section{Practice and Conjunction Search}

Performance on most tasks improves with practice, and visual search is no exception. Schneider and Shiffrin (1977) demonstrated that large display size effects could be eliminated when targets and distractors were consistently mapped to responses over several thousand practice trials. This practice-based change in the attentional demands of the task has been reported for conjunctions of contrast polarity and shape (Theeuwes \& Kooi, 1994), brightness and orientation (Nakayama \& Mackeben, 1989), and some combinations of stereopsis, orientation, vernier offset, and lateral separation (S. B. Steinman, 1987). Finally, Friedman-Hill and Wolfe (1995) found that display size effects were halved in as few as 1,000 conjunction search trials.

The mechanisms responsible for these changes are a matter of debate. It may be that efficient conjunction search results from parallel processing of groups of items, as has been proposed by Treisman and Gormican (1988) for difficult feature search. Zohary and Hochstein (1989) argued that for color $\times$ orientation search, groups of 6-16 elements are processed every $50-150 \mathrm{msec}$. Alternatively, observers may select a relevant subset (e.g., all red items) and then search for the "odd man out" in that subset (Friedman-Hill \& Wolfe, 1995; Kaptein, Theeuwes, \& van der Heijden, 1995; Kim \& Cave, 1995). This explanation is tantamount to suggesting that observers convert the task to one of feature search and, once it is converted, they show the expected minimal display size effects.

If observers select items on the basis of their features, and if the oculomotor and attentional systems are linked, one would expect to see evidence of selectivity in fixation data. It is surprising, therefore, that Zelinsky (1996) reported that observers' fixations are no more likely to fall on elements sharing one target feature as to fall on elements sharing no feature with the target. There are several reasons to think that Zelinsky's data need replication. Observers were unpracticed and, as noted, selection may be based on experience. Additionally, the critical condition in which some distractors were made dissimilar to the target also increased distractor heterogeneity along the relevant dimensions for the task (i.e., orientation and color). As such, efficient selection may have been more difficult (Duncan \& Humphreys, 1989). Finally, in Zelinsky's study, observers found the target within two saccades on more than $65 \%$ of the trials. Under these conditions, it would be difficult to find evidence of selectivity in the fixation data.

\section{EXPERIMENT 1A}

In Experiment 1A, observers were asked to detect a target defined by a unique relative contrast or orientation value and also to detect targets defined as the conjunction of two feature values along these dimensions. This was a present-absent task in which target eccentricity varied. It was expected that for feature search, both RT and number of saccades would be relatively independent of eccentricity. For conjunction search, however, it was expected that RTs would be longer and saccades more frequent, particularly when targets were presented at more eccentric locations. Predictions about average fixation duration and eccentricity were not made because this was a free-viewing procedure and it is unclear why fixation duration would change as a function of target location once an element had been fixated.

\section{Method}

Participants. Six young adults participated in Experiment 1A, all of whom worked in the authors' lab and were experienced with search tasks. They had normal or corrected acuity and wore their presenting corrections during testing.

Materials and Apparatus. The stimuli for all conditions consisted of lines measuring $0.64^{\circ}$ in length and $0.13^{\circ}$ in width. These elements were placed within the cells of an imaginary matrix $30.5^{\circ}$ high $\times 23.5^{\circ}$ wide at the viewing distance of $45 \mathrm{~cm}$. Display size was $72 \pm 2$ elements. Targets were placed along the diagonals at eccentricities of $3.82^{\circ}, 8.26^{\circ}$, and $13.94^{\circ}$. Within each cell, elements were randomly jittered $.25^{\circ}$ to eliminate luminance cues (Nothdurft, 1985). Minimal and maximal line separation was $1.27^{\circ}$ and $5.22^{\circ}$, respectively.

In the feature orientation condition, the target was a black line oriented $45^{\circ}$ to the right of vertical. It was embedded in black distractors oriented $45^{\circ}$ to the left. Line luminance was $.91 \mathrm{~cd} / \mathrm{m}^{2}$ and background luminance was $47.36 \mathrm{~cd} / \mathrm{m}^{2}$; thus Michelson contrast 
was $97 \%$. In the feature contrast condition, the target was a white line oriented $45^{\circ}$ to the right. Distractors were black lines of the same orientation. Target luminance was approximately $78.21 \mathrm{~cd} / \mathrm{m}^{2}$; its contrast was $25 \%$. In the conjunction condition, the target was defined as a white line oriented $45^{\circ}$ to the right. It was embedded in approximately equal numbers $(35 \pm 2)$ of black lines oriented $45^{\circ}$ to
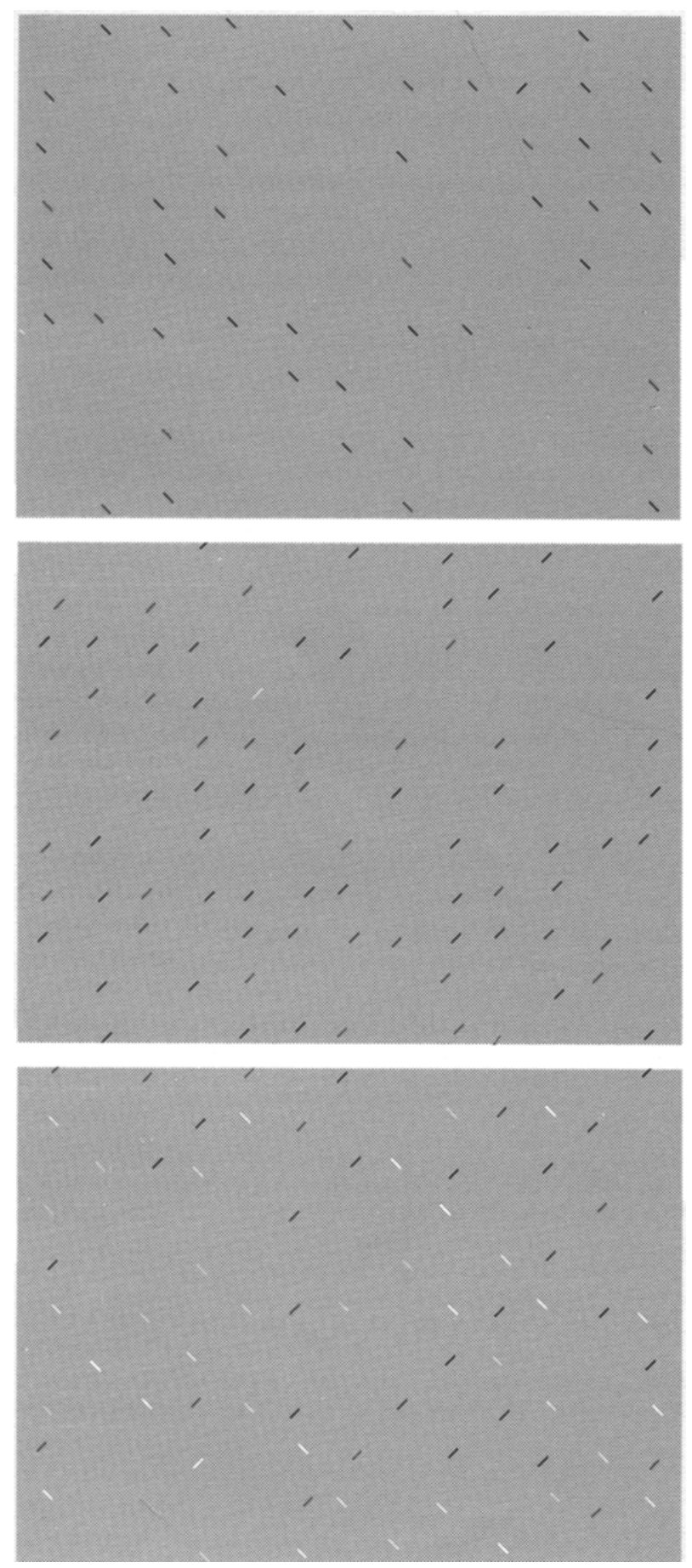

Figure 1. Examples of displays used in Experiment 1A. Top panel: feature orientation. Middle panel: feature contrast. Bottom panel: conjunction. the right and white lines oriented $45^{\circ}$ to the left. Examples of displays from each search condition are shown in Figure 1.

Search displays were presented and data collected using the Eyegaze Development System and software from LC Technologies, Inc. (Fairfax, VA: Cleveland \& Cleveland, 1992). The system uses an 80486 computer platform to generate the stimuli and record responses. Displays were presented on a Panasonic PanaSync C1381i VGA Monitor. Eye movements were sampled at $30.3 \mathrm{~Hz}$ using the pupil center/corneal reflection technique (Young \& Sheena, 1975). Spatial resolution was better than $.5^{\circ}$.

A height-adjustable chinrest and chair, along with a forehead restraint, were used to keep the observer's head in a fixed position at a constant distance from the monitor and at a vertical gaze angle of approximately $0^{\circ}$ with respect to the fixation stimulus.

Procedure. Participants were tested in a single session lasting approximately $90 \mathrm{~min}$. General information pertaining to the task was supplied, including a description of display elements and the appropriate keypress response ("C" key for absent with left hand and " $M$ " key for present with right hand). Both speed and accuracy were emphasized, but participants were instructed to slow their responses if they made more than two errors per block of 36 trials.

Each trial began with a fixation point $1.27^{\circ}$ in diameter, having a luminance of $0.91 \mathrm{~cd} / \mathrm{m}^{2}$. After fixating this stimulus, observers pressed any key to begin the trial. The fixation stimulus disappeared immediately and the search display was presented $130 \mathrm{msec}$ later, remaining visible until a response was made. Accuracy feedback was provided (+ for correct, - for incorrect) for $1 \mathrm{sec}$, after which the predisplay fixation point reappeared.

The experiment consisted of 432 trials, 144 in each of three search conditions. Within each search condition, there were 72 target-present and target-absent trials and, within the target-present trials, there were 24 trials at each of the three levels of eccentricity. Trials were separated into four blocks of 36 trials each. Observers were given a brief rest period after each two blocks.

Target presence and eccentricity were randomized within each block with the restriction that there was an equal number of trials for each target location. Order of search condition was counterbalanced across participants.

\section{Results}

Accuracy. The effects of search condition and eccentricity on accuracy on target-present trials were analyzed in a search condition (3) $\times$ eccentricity (3) univariate repeated measures analysis of variance (ANOVA). A separate analysis was conducted to determine the effects of target presence because eccentricity was irrelevant on target-absent trials.

Overall accuracy was high for both the feature orientation $(98.1 \%)$ and feature contrast $(95.4 \%)$ conditions but was reduced to $84.5 \%$ in conjunction search. This difference in accuracy was reflected in the significant main effect of search condition $[F(2,10)=8.48, p=.007]$.

The main effect of eccentricity was also significant $[F(2,10)=19.90, p<.001]$. As Figure 2 indicates, accuracy was reduced at more eccentric target locations in all viewing conditions. However, this eccentricity effect was largest in conjunction search, supported by a significant search condition $\times$ eccentricity interaction $[F(4,20)=$ $4.63, p=.008]$. Follow-up tests indicated that the eccentricity effect was obtained in conjunction search only.

Conjunction search also yielded the largest effect of target presence. A search condition (3) $\times$ presence (2) ANOVA revealed significant main effects of search con- 

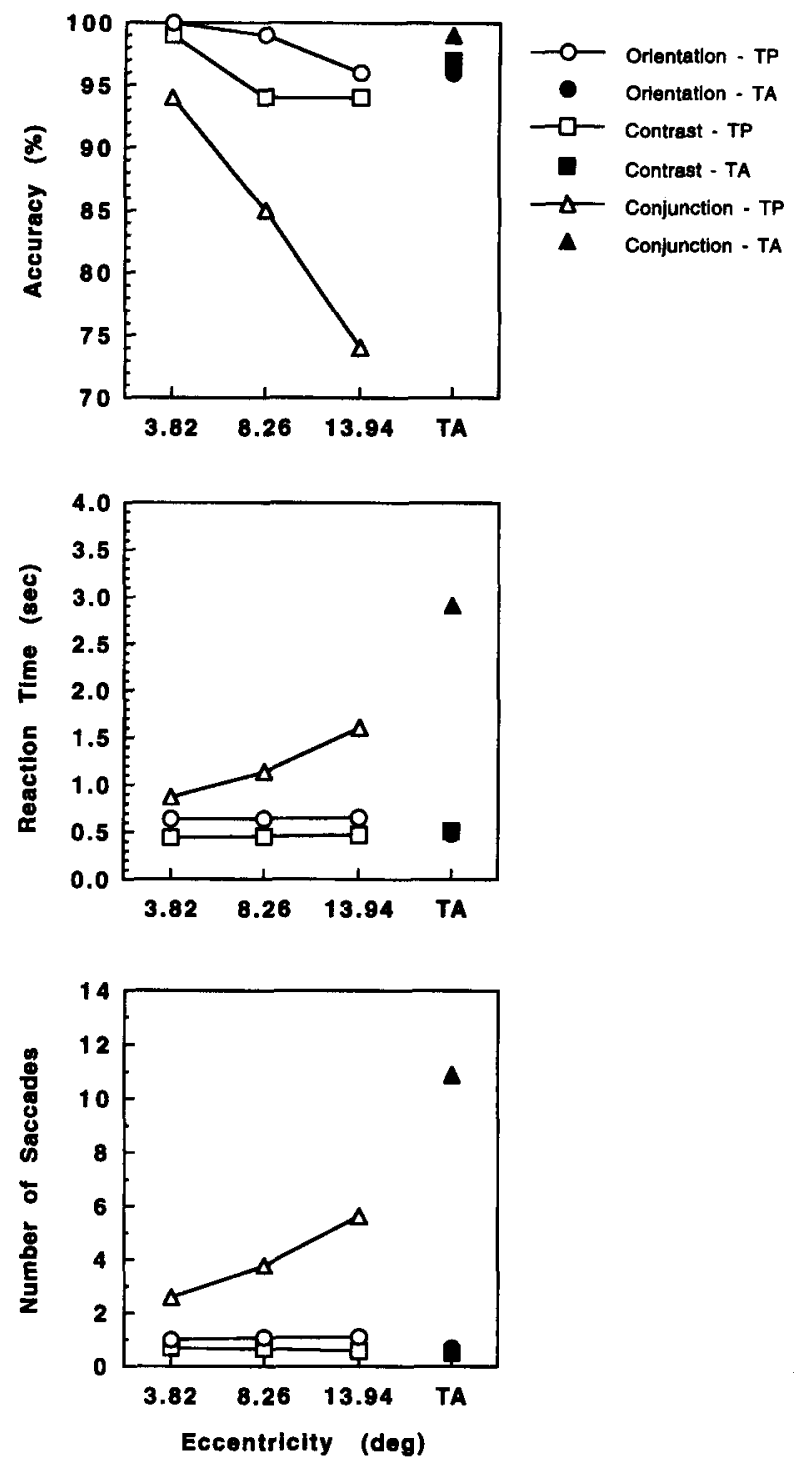

Figure 2. Experiment 1A. Search performance as a function of eccentricity, target presence, and search condition. Top panel: percent accuracy. Middle panel: reaction time. Bottom panel: number of saccades.

dition $[F(2,10)=5.42, p=.025]$ and presence $[F(1,5)=$ $8.93, p=.03]$, as well as a search condition $\times$ presence interaction $[F(2,10)=8.87, p=.006]$. Follow-up tests indicated that the presence effect was obtained in conjunction search only. All subsequent analyses were conducted only for trials ending in a correct response.

Reaction times. Figure 2 also shows that RTs clearly increased in conjunction search. RTs were slowed considerably for more eccentric target presentations in the conjunction condition, but were independent of eccentricity when targets were defined by orientation or contrast alone. Again, the greatest effect of target presence was found in conjunction search.

A search condition $(3) \times$ eccentricity (3) ANOVA revealed main effects of search condition $[F(2,10)=38.00$, $p<.001]$ and eccentricity $[F(2,10)=26.77, p<.001]$, as well as a search condition $\times$ eccentricity interaction $[F(4,20)=19.07, p=.002]$. Follow-up tests indicated that an eccentricity effect was obtained in conjunction search only. A search condition (3) $\times$ presence (2) ANOVA revealed significant effects of presence $[F(1,5)=23.56$, $p=.005]$ and search condition $[F(2,10)=39.74, p<$ $.001]$. A significant search condition $\times$ presence interaction was also found $[F(2,10)=29.58, p<.001]$, reflecting the fact that RTs were increased greatly on targetabsent trials in conjunction search, which was confirmed by follow-up tests.

Saccades. Number of saccades was determined by visual inspection of the scan paths interposed on the displays. Generally, at least two samples in a location different from the current fixation were required to record an eye movement. If a number of samples were taken at perceptibly different locations, but all were clustered within a small area, no additional movement was recorded. If a single sample was taken between two clusters, it was not typically considered a saccade. However, if a large change in the direction of the scan path was separated by a single sample, two saccades were recorded. This procedure, when used by two raters, yielded an interrater reliability in excess of .95 for 360 displays seen for 5 randomly chosen participants.

As shown in Figure 2, a pattern similar to that of RTs emerged when the saccade data were analyzed. The greatest number of saccades, and the only measurable effect of target eccentricity, was found in the conjunction condition. In addition, although target presence had little effect in feature search, saccades were more frequent on targetabsent trials in the conjunction condition.

These trends were corroborated in a search condition (3) $\times$ eccentricity (3) ANOVA. The main effects of search condition $[F(2,10)=51.12, p<.001]$ and eccentricity $[F(2,10)=22.14, p<.001]$ were found to be significant, as was the search condition $\times$ eccentricity interaction $[F(4,20)=13.14, p<.001]$. Tests of simple effects indicated that the effect of eccentricity was significant only in conjunction search. The search condition (3) $X$ presence $(2)$ analysis revealed a significant interaction $[F(2,10)=62.15, p<.001]$, along with main effects of presence $[F(1,5)=28.80, p=.003]$ and condition $[F(2,10)=64.24, p<.001]$. Follow-up tests indicated that there was a significant presence effect only in conjunction search.

Average fixation duration. Average fixation durations are shown in Table 1. In several ways, the trends are the reverse of those found in the RT, accuracy, and saccade data. Target-absent trials resulted in shorter fixations than target-present trials in feature orientation and conjunction search, but not in feature contrast. The effect of eccentricity was minimal for conjunction search, but substantial in feature contrast displays. Further, conjunction search was associated with the shortest fixation durations.

A search condition (3) $\times$ eccentricity (3) ANOVA on target-present trials revealed no main effect of eccentricity $(p=.87)$ but did reveal a main effect of search con- 
Table 1

\begin{tabular}{|c|c|c|c|c|c|c|c|c|c|c|c|c|c|c|c|c|}
\hline \multirow[b]{5}{*}{ Condition } & \multicolumn{8}{|c|}{ Experiment $1 \mathrm{~A}$} & \multicolumn{8}{|c|}{ Experiment 1B } \\
\hline & \multicolumn{6}{|c|}{$\mathrm{TP}$} & & & \multicolumn{6}{|c|}{ TP } & & \\
\hline & \multicolumn{6}{|c|}{ Eccentricity } & & & \multicolumn{6}{|c|}{ Eccentricity } & & \\
\hline & \multicolumn{2}{|c|}{$3.82^{\circ}$} & \multicolumn{2}{|c|}{$8.26^{\circ}$} & \multicolumn{2}{|c|}{$13.94^{\circ}$} & \multicolumn{2}{|c|}{ TA } & \multicolumn{2}{|c|}{$3.82^{\circ}$} & \multicolumn{2}{|c|}{$8.26^{\circ}$} & \multicolumn{2}{|c|}{$13.94^{\circ}$} & \multicolumn{2}{|c|}{ TA } \\
\hline & $M$ & $S D$ & $M$ & $S D$ & $M$ & $S D$ & $M$ & $S D$ & $M$ & $S D$ & $M$ & $S D$ & $M$ & $S D$ & $M$ & $S D$ \\
\hline \multicolumn{17}{|l|}{ Feature orientation } \\
\hline Average fixation & 380 & 119 & 382 & 126 & 378 & 112 & 327 & 93 & 343 & 171 & 331 & 174 & 306 & 150 & 309 & 108 \\
\hline First fixation & 416 & 106 & 426 & 103 & 425 & 84 & 342 & 86 & 404 & 148 & 378 & 134 & 366 & 122 & 317 & 109 \\
\hline \multicolumn{17}{|l|}{ Feature contrast } \\
\hline Average fixation & 314 & 89 & 326 & 99 & 344 & 110 & 410 & 72 & 364 & 126 & 380 & 136 & 377 & 125 & 318 & 91 \\
\hline First fixation & 331 & 93 & 341 & 94 & 361 & 102 & 433 & 80 & 391 & 98 & 410 & 118 & 413 & 92 & 315 & 95 \\
\hline \multicolumn{17}{|l|}{ Conjunction } \\
\hline Average fixation & 255 & 46 & 235 & 30 & 228 & 23 & 218 & 14 & 223 & 32 & 208 & 35 & 196 & 14 & 190 & 11 \\
\hline First fixation & 325 & 50 & 315 & 33 & 321 & 56 & 313 & 44 & 243 & 22 & 243 & 36 & 235 & 31 & 249 & 17 \\
\hline
\end{tabular}

TP, target present; TA, target absent.

dition $[F(2,10)=5.08, p=.03]$. The search condition $X$ eccentricity interaction was significant $[F(4,20)=$ $3.26, p=.033$ ], but follow-up tests indicated that the eccentricity effect was nonsignificant in all search conditions. The search condition (3) $\times$ presence (2) ANOVA yielded a nonsignificant effect of presence $(p=.78)$, but a significant effect of search condition $[F(2,10)=7.89$, $p=.009]$. The significant search condition $\times$ presence interaction $[F(2,10)=16.69, p=.001]$ arose because target-absent durations were significantly shorter than target-present durations in conjunction and feature orientation displays, but significantly longer in feature contrast displays.

First fixation duration. Also shown in Table 1 are the mean first fixation durations for each condition, which were significantly longer than the average fixation durations $[F(1,5)=36.83, p=.002]$. As with average fixation durations, there was a trend for first fixations to be shorter for target-absent trials and conjunction search displays.

A search condition (3) by eccentricity (3) ANOVA on target-present trials revealed no effect of eccentricity $(p=.161)$, but did reveal a main effect of search condition $[F(2,10)=4.25, p=.046]$, wherein the durations for conjunction search were significantly shorter than the durations for feature search. The search condition $x$ eccentricity interaction was nonsignificant $(p=.355)$. The search condition ( 3$) \times$ presence $(2)$ ANOVA yielded a nonsignificant effect of presence $(p=.943)$ and search condition $(p=.109)$, but a search condition $\times$ presence interaction $[F(2,10)=17.65, p=.001]$ arose because the first fixations were independent of target presence in conjunction search and were significantly shorter on targetpresent trials in feature contrast, but were significantly shorter for target-absent trials in feature orientation.

Relation between number of saccades and fixation duration. These seemingly counterintuitive findings would make sense if fixation durations and number of saccades were related inversely to each other, as would be expected if the information processed per fixation were reduced when there are many fixations (and thus, many saccades). Reexamination of the saccade data provides some evidence in favor of this hypothesis. There was a larger number of saccades for more eccentric targets in conjunction search, especially on target-absent trials, the conditions often associated with shorter fixation durations.

To explore this possibility further, both linear and power functions were fitted to the individual data relating average fixation duration and saccade number. The data were collapsed across eccentricity and target presence because we needed to maximize the number of trials used to estimate fixation duration at each level of saccade number. An ANOVA revealed that the power functions accounted for significantly more variance than the linear functions in all search conditions, so only results from the power function analyses will be reported.

On average, power functions accounted for $77 \%$ of the variance in feature orientation data, $90 \%$ of the variance in feature contrast data, and $69 \%$ of the variance in conjunction search data. Shown in Figure 3 are curves for the three search conditions, for which the data points were calculated from group averages of the power function parameters (hence the lack of variance about the functions). The average intercept was larger for conjunction search $(M=317 \mathrm{msec})$ than for feature orientation $(M=$ $306 \mathrm{msec})$ and feature contrast $(M=249 \mathrm{msec})$. Analysis of the exponents indicated that the decrease in average fixation associated with more saccadic involvement was not greatly different for conjunction search $(M=$ $-.24)$ than for feature orientation $(M=-.26)$ or feature contrast $(M=-.33)$. ${ }^{1}$

Rechecking. It has been suggested that the greater display size effects found in conjunction displays arise because of a greater tendency to recheck the display (Plude \& Doussard-Roosevelt, 1989). Therefore, we also determined the frequency with which an observer's gaze returned to a previously fixated area. This was accomplished 

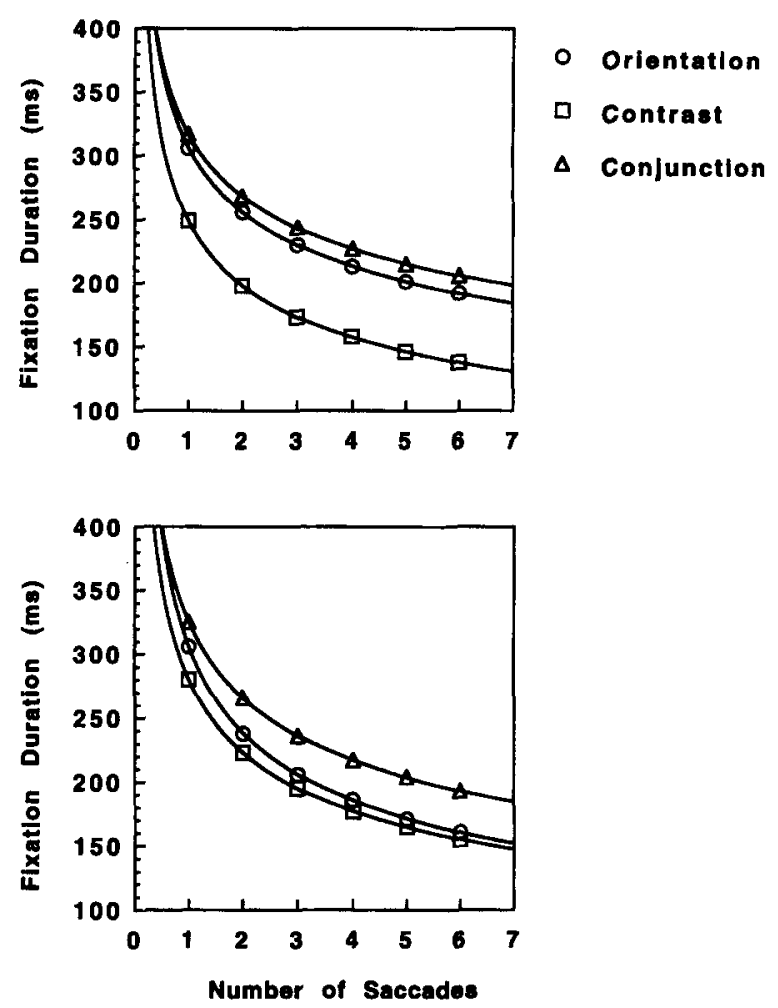

Figure 3. Power functions relating average fixation duration and number of saccades. Top panel: Experiment 1A. Bottom panel: Experiment $1 B$.

by superimposing an $11 \times 13$ cell grid on the displays, each cell measuring $2.39^{\circ}$ high $\times 2.16^{\circ}$ wide. A recheck was said to occur if two consecutive samples fell in a cell that had previously been searched. Rechecking occurred with extremely low frequency for all observers (range $=$ $0-.30$ fixations per trial). Even in the most difficult search conditions a region was rechecked only once every three trials. For this reason, the data are not shown.

On average, the number of rechecks was elevated slightly, and the greatest effect of target presence was found in the conjunction search condition. The only meaningful results of the eccentricity $(3) \times$ search condition (3) ANOVA was the significant main effect of search condition $[F(2,10)=18.18, p=.012]$. The presence $\times$ search condition ANOVA revealed main effects of search condition $[F(2,10)=9.93, p=.004]$ and presence $[F(1,5)=5.56, p=.065]$, along with a search condition $\times$ presence interaction $[F(2,10)=8.09, p=.008]$; rechecking was more likely on target-absent, conjunction search trials.

\section{Discussion}

The results of Experiment $1 \mathrm{~A}$ are consistent with the view that feature search is independent of eccentricity. Observers had little difficulty detecting feature search targets defined by their unique orientation or relative contrast.
Further, observers averaged no more than one eye movement prior to target detection, regardless of eccentricity.

In contrast, accuracy declined significantly, RTs increased in duration, and number of saccades increased for more eccentric conjunction search trials. However, even for the most eccentric locations, fewer than six saccades on average were required for detection of the target, approximately half the number executed on target-absent trials. These data might be explained by arguing that observers conduct a parallel search of small groups of items (Pashler, 1987; Poisson \& Wilkinson, 1992; Treisman \& Gormican, 1988; Zohary \& Hochstein, 1989). Alternatively, it may be that attention is guided to individual items, or perhaps subsets of items, on the basis of relative activation levels (Cave \& Wolfe, 1989; Wolfe, 1994; Wolfe, Cave, \& Franzel, 1989), thus increasing efficiency compared with a completely random, selfterminating, serial search. Either model could explain why highly discriminable conjunction targets can yield parallel search functions (McLeod, Driver, \& Crisp, 1988; Nakayama \& Silverman, 1986).

On the surface, both the first fixation and the average fixation duration data are troubling because the conditions generally associated with longer RTs, lower accuracy, and a larger number of saccades (e.g., conjunction search) also produced the shortest fixation durations. The power function analyses help explain why this is so. For all conditions, fixation durations declined in a nonlinear manner when saccades were more numerous. Examination of Figure 3 indicates that, for a constant number of saccades, fixation duration tends to be longer in conjunction search, where processing is assumed to be more demanding. These relations would be expected if observers were searching displays in groups of variable size and the time taken to process the group was related to the size and featural complexity of the group.

It has been suggested that participants recheck items on target-absent trials (Plude \& Doussard-Roosevelt, 1989; Zacks \& Zacks, 1993), and that as a result, targetabsent and target-present RTs have a ratio greater than $2: 1$. Rechecking of previously attended locations has been found to be rare in feature search (Scialfa et al., 1994) and does not appear to play a large role in conjunction search, either. On target-present trials, rechecking occurred on less than $15 \%$ of trials, and even on target-absent displays, rechecks occurred less than once every three trials. However, this conclusion must be qualified because if observers are searching groups of items, the rechecking analysis conducted may not reveal those fixations containing partially overlapping areas.

\section{EXPERIMENT 1B}

The first experiment revealed that conjunction displays impose substantial attentional and oculomotor demands on the observer. It is important to recall, however, that the feature and conjunction tasks used differed in within- 
trial distractor variability. There was only one distractor type in each feature search condition, but two distractor types in the conjunction displays. Duncan and Humphreys (1989) have discussed the importance of distractor heterogeneity, and although Treisman (1988) reported that feature search RTs were largely unaffected by distractor heterogeneity along an "irrelevant dimension," we wanted to determine whether this finding would be obtained with eye movement data. So, in the next experiment, we conducted a substantive replication of Experiment $1 \mathrm{~A}$, but held constant the number of different types of distractors uses in each search condition.

\section{Method}

Participants. Six young adult University of Calgary students participated in Experiment 1B. They had no previous experience with laboratory search tasks and were paid $\$ 10$ (Canadian) for their participation. All observers had normal or corrected-to-normal acuity when refracted for the test distance of $45 \mathrm{~cm}$.

Materials, Apparatus, and Procedure. Experiment $1 \mathrm{~B}$ was identical to Experiment $1 \mathrm{~A}$ but for the following changes. To avoid the possibility of negative transfer when participants respond to black and then white targets (Schneider \& Shiffrin, 1977), in all search conditions, the target was a white line oriented $45^{\circ}$ to the right. In
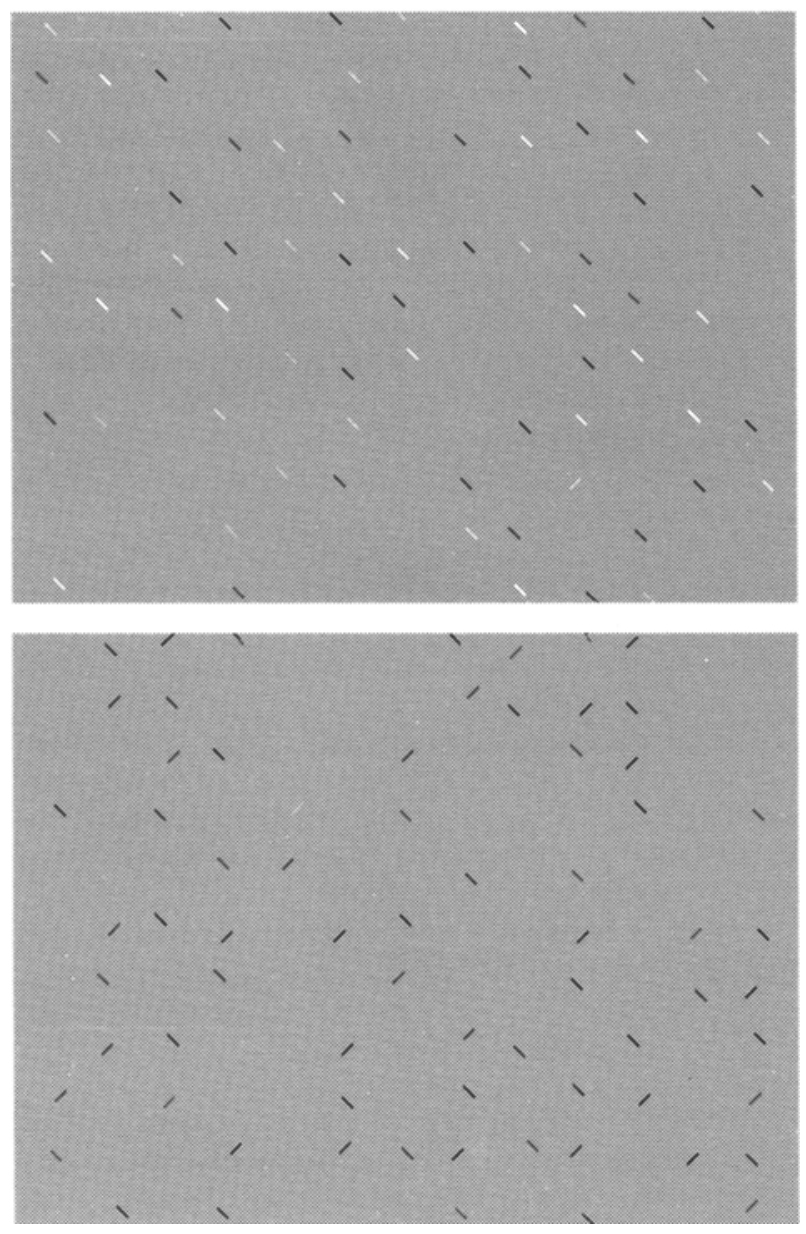

Figure 4. Examples of displays used in Experiment 1B. Top panel: feature orientation. Bottom panel: feature contrast.
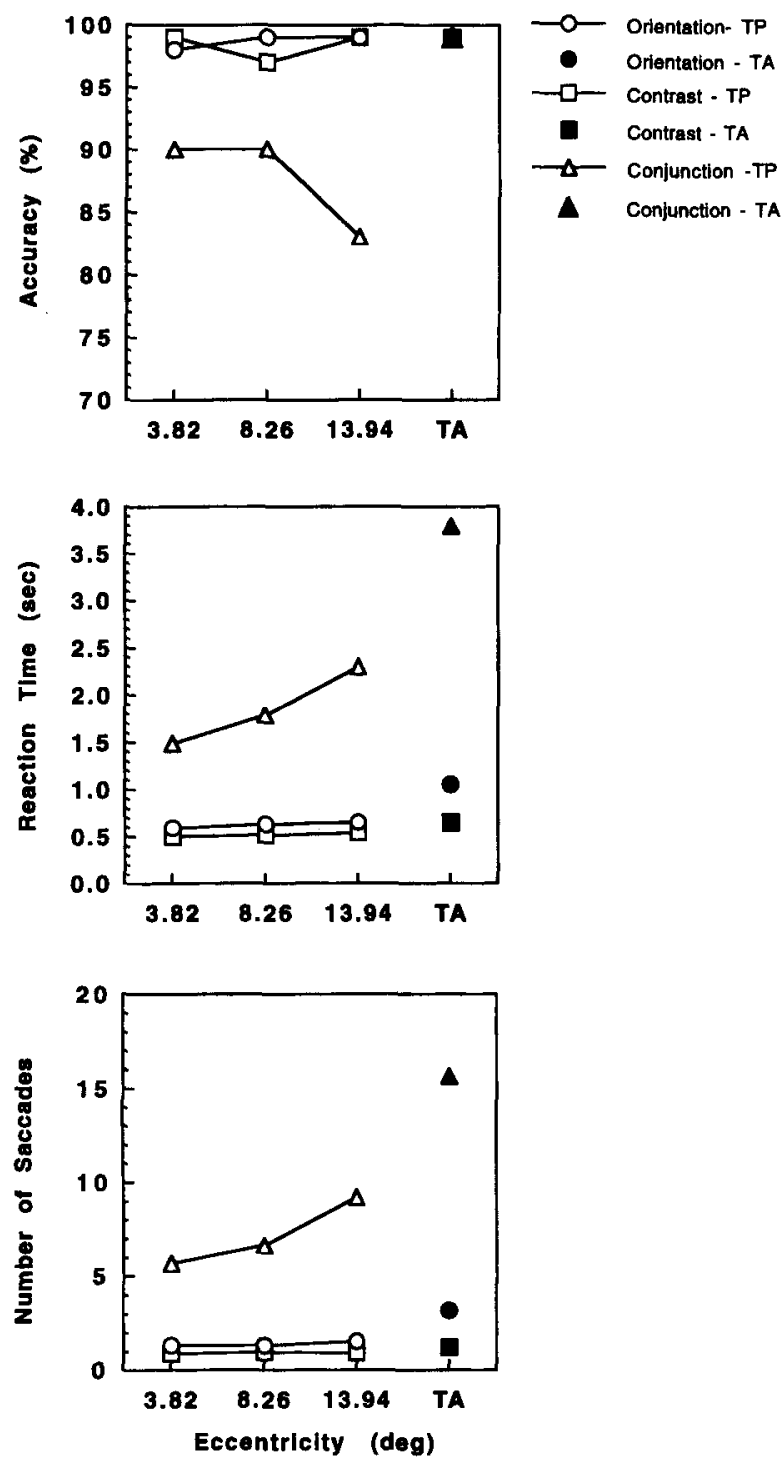

Figure 5. Experiment 1B. Search performance as a function of eccentricity, target presence, and search condition. Top panel: percent accuracy. Middle panel: reaction time. Bottom panel: number of saccades.

the feature orientation condition, distractors were white lines and black lines oriented $45^{\circ}$ to the left. In the feature contrast condition, distractors were black lines oriented $45^{\circ}$ left and right. Conjunction search displays were unchanged. Examples of displays from the feature orientation and feature contrast conditions are shown in Figure 4.

\section{Results}

Accuracy. As shown in Figure 5, target identification accuracy in both the feature orientation $(98.6 \%)$ and the feature contrast conditions $(98.1 \%)$ approached ceiling. Accuracy in conjunction search was reduced in comparison $(87.5 \%)$, particularly when targets were present at the most eccentric locations. These relations were analyzed in a search condition (3) $\times$ eccentricity (3) ANOVA and a separate analysis to determine the effects of target presence. 
The main effect of search condition was significant $[F(2,10)=10.09, p=.004]$, but the main effect of eccentricity was not $(p=.186)$. There was a marginally significant search condition $\times$ eccentricity interaction $[F(4,20)=2.84, p=.051]$, but the eccentricity effect was nonsignificant in any single search condition.

A search condition (3) $\times$ presence (2) ANOVA revealed main effects of search condition $[F(2,10)=6.94$, $p=.013]$ and presence $[F(1,5)=14.97, p=.012]$, as well as the search condition $\times$ presence interaction $[F(2,10)=$ $13.92, p<.001]$. Follow-up tests revealed a significant presence effect only in conjunction search.

Reaction times. RTs, depicted in Figure 5, were slowest in the conjunction search condition, and, although they changed minimally across eccentricity in both the feature search conditions, they were slowed by approximately $800 \mathrm{msec}$ for more eccentric target presentations in conjunction search. As in Experiment $1 \mathrm{~A}$, the largest effect of target presence was found in conjunction search, but here this effect was also evident in the feature orientation condition.

These results were substantiated in a search condition (3) $\times$ eccentricity (3) ANOVA. The main effects of search condition $[F(2,10)=8.89, p=.006]$ and eccentricity $[F(2,10)=16.30, p<.001]$ were significant, as was their interaction $[F(4,20)=12.95, p=.001]$. Simple effects tests revealed significant or marginally significant effects of eccentricity in all search conditions. As shown in Figure 5, the interaction arose because the effect was much larger in conjunction search than in either feature search condition, where the eccentricity effect was less than $6 \mathrm{msec}$ per degree of visual angle. The search condition (3) $\times$ presence (2) ANOVA revealed significant search condition $[F(2,10)=10.22, p=.004]$, presence $[F(1,5)=28.99, p=.003]$, and search condition $\times$ presence effects $[F(2,10)=11.78, p=.002]$. Only in conjunction search was the presence effect significant.

Saccades. The eye movement data, shown in Figure 5, indicated that the greatest effects of target eccentricity and presence were found in conjunction search. These trends were seen in the ANOVAs. Both the main effects of search condition $[F(2,10)=15.19, p=.011]$ and eccentricity $[F(2,10)=8.18, p=.008]$ were found to be significant, along with the search condition $\times$ eccentricity interaction $[F(4,20)=10.04, p<.001]$. Only in conjunction search was the eccentricity effect significant. The search condition ( 3$) \times$ presence $(2)$ ANOVA revealed significant main effects of search condition $[F(2,10)=$ $14.43, p=.001]$ and presence $[F(1,5)=28.73, p=.003]$. A significant search condition $x$ presence interaction was also detected $[F(2,10)=12.41, p=.002]$. As expected, the presence effect was significant only in conjunction search.

Average fixation duration. Table 1 displays the average fixation data as a function of eccentricity, presence, and search condition. Again, in several ways the trends are the reverse of those found for RTs and number of sac- cades. Average fixations were shorter for more eccentric targets, target-absent trials, and conjunction search.

An eccentricity (3) $\times$ search condition (3) ANOVA revealed that the effects of eccentricity $(p=.115)$ and eccentricity by search condition $(p=.091)$ were nonsignificant. A main effect of search condition was obtained $[F(2,10)=5.94, p=.02]$. The search condition (3) $\times$ presence (2) ANOVA yielded a significant main effect of condition $[F(2,10)=7.19, p=.012]$. Feature search resulted in significantly longer average fixations than did conjunction search. Neither the main effect of presence $(p=.102)$ nor the search condition $\times$ presence interaction $(p=.208)$ were significant.

First fixation duration. Also shown in Table 1 are the mean first fixation durations for each condition. As in Experiment $1 \mathrm{~A}$, first fixations were significantly longer than average fixations $[F(1,5)=17.73, p=.008]$ and tended to be shorter on target-absent trials and in conjunction search displays.

An eccentricity (3) $\times$ search condition (3) ANOVA revealed that the effect of eccentricity ( $p=.574)$ was nonsignificant. However, there was a main effect of search condition $[F(2,10)=7.99, p=.008]$ and a search condition by eccentricity interaction $[F(4,20)=5.64, p=$ $.003]$. Only in feature orientation was there a significant eccentricity effect, where first fixation duration was shorter for more eccentric targets. The search condition (3) $\times$ presence (2) ANOVA yielded a significant main effect of search condition $[F(2,10)=5.13, p=.029]$. The main effect of presence was significant $[F(1,5)=$ $47.56, p<.001]$, as was the search condition $\times$ presence interaction $[F(2,10)=18.15, p<.001]$. In both feature search conditions, first fixations were significantly shorter for target-absent trials. There was no presence effect in conjunction search.

Relation between number of saccades and fixation duration. Both linear and power functions were fit to the data relating average fixation duration and saccade number. Power functions accounted for significantly more variance in all search conditions, so only these results will be reported. Data from 2 observers were omitted; we could not estimate parameters of their functions due to too few saccades.

On average, power functions accounted for $79 \%$ of the variance in feature orientation data, $89 \%$ of the variance in the feature contrast data, and $74 \%$ of the variance in the conjunction search data. Shown in Figure 3 are curves for the three search conditions; the data points were calculated from group averages of the power function parameters. The average intercept was greater for conjunction search $(M=325 \mathrm{msec})$ than for feature orientation $(M=306 \mathrm{msec})$ or feature contrast $(M=280 \mathrm{msec})$. Analysis of the exponents indicated that the decrease in average fixation associated with more saccadic involvement was approximately equal for conjunction search $(M=-.29)$, feature orientation $(M=-.36)$, and feature contrast $(M=-.33) .^{2}$ 
Rechecking. Rechecking occurred with low frequency, ranging from .009 to .964 . In feature search, rechecks were made about once per 20 trials. In conjunction search, rechecks occurred approximately once per target-absent trial and once every other target-present trial. ANOVAs revealed that the effects of presence $(p=.088)$ and search condition $(p=.090)$, as well as their interaction $(p=$ .062 ), all approached significance, but due to the low probability of rechecking, these data will not be discussed further.

\section{Discussion}

Consistent with Treisman (1988), Experiment 1B showed that the feature search advantage does not occur trivially because of distractor homogeneity. As found by Scialfa et al. (1994), when feature search displays contained two distractor types, their feature values varying on an irrelevant dimension, search was significantly related to target eccentricity. However, eccentricity produced a much larger effect on conjunction search. It is unlikely, then, that the conjunction search deficits seen in Experiments $1 \mathrm{~A}$ and $1 \mathrm{~B}$ were caused by distractor heterogeneity.

As in Experiment 1A, relative to feature search, conjunction search yielded longer RTs, more errors, and a greater number of saccades, particularly for eccentric targets. Feature search was much less dependent on eccentricity. Again, the conjunction search condition was generally associated with shorter fixation durations, and power functions provided a reasonable fit to the data relating the number of saccades and fixation duration.

Across Experiments $1 \mathrm{~A}$ and $1 \mathrm{~B}$, the power functions relating fixation duration to the number of saccades accounted for approximately $80 \%$ of the variance in fixation duration data. A second approach to examining the relation between number of saccades and fixation duration is to directly compare fixation durations in those trials in which the number of saccades was held constant. A limitation of this approach, suggested by Figures 2 and 5 , is that there were few instances in which equal numbers of saccades were made in all search conditions. However, across both experiments there were 10 observers who, in all search conditions, provided reliable fixation duration data for trials ending after either one or two saccades.

The mean fixation durations on trials ending in one saccade were 305,256 , and $356 \mathrm{msec}$ for feature orientation, feature contrast, and conjunction search, respectively. The comparable means for trials ending in two saccades were 225,195 , and $260 \mathrm{msec}$. An ANOVA indicated that there was a significant main effect of search condition for the "one-saccade" data $[F(2,18)=4.67$, $p=.023]$ and the "two-saccade" data $[F(2,18)=5.60$, $p=.013]$. For both measures, the fixation durations associated with conjunction search were significantly longer than those associated with the feature search conditions considered together $(p<.05)$. Additionally, in all three search conditions, the fixation durations associated with one-saccade trials were significantly longer than those associated with two-saccade trials. These tests complement the power function analyses in showing that fixa- tion duration is a nonlinearly decreasing function of the number of saccades; in addition, however, they indicate that fixation duration is longer for conjunction than for feature search when comparable trials are considered (Scialfa \& Joffe, 1997). ${ }^{3}$

\section{EXPERIMENT 2}

Experiments $1 \mathrm{~A}$ and $1 \mathrm{~B}$ showed that conjunction search has large oculomotor demands, but some data suggest that saccades are unnecessary for search. Klein and Farrell (1989) found that display size effects in feature search do not depend on eye movements, even when serial search functions are produced. More importantly, Nakayama and Mackeben (1989) reported that observers did not need to make eye movements to identify a search target defined by the conjunction of brightness and orientation. In Experiment 2, therefore, participants were required to search for a conjunction target when eye movements were precluded. If performance declines in comparison to that in Experiments $1 \mathrm{~A}$ and 1B, one can assert that eye movements are functional and reflect in part the differing demands of feature and conjunction search.

\section{Method}

Participants. Six young adult University of Calgary students with no previous experience in laboratory search tasks participated
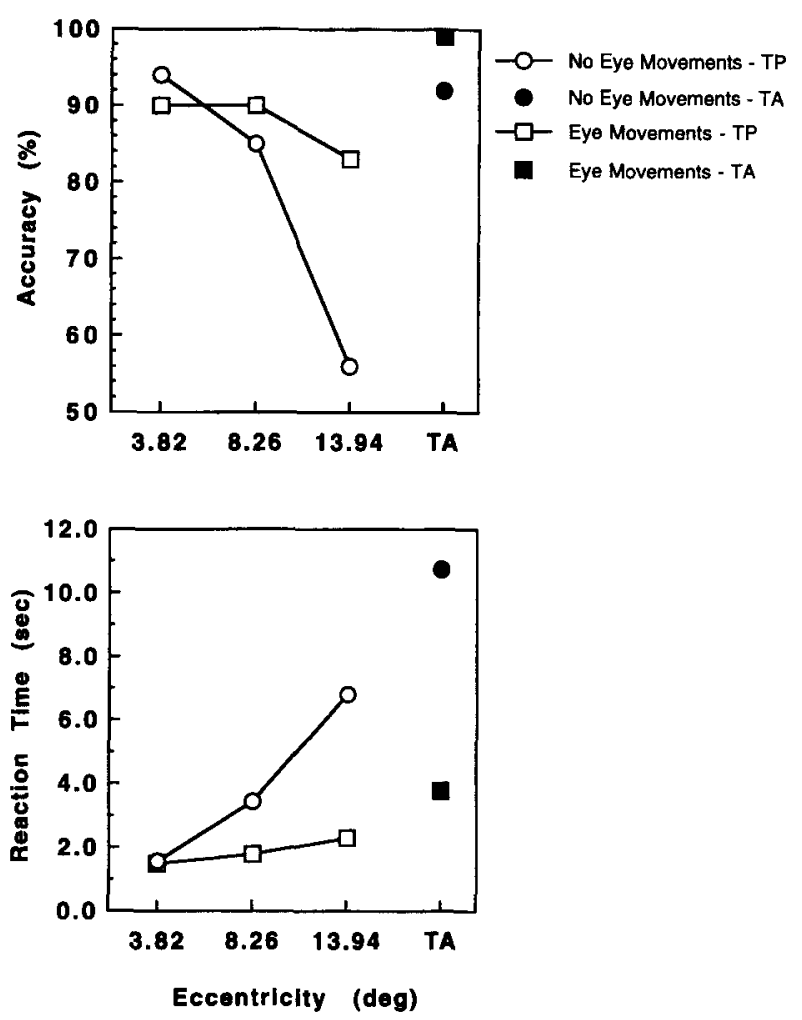

Figure 6. Experiment 2. Search performance as a function of eccentricity, target presence, and eye movements, replotted in part from Experiment 1 B. Top panel: percent accuracy. Bottom panel: reaction time. 
and were paid $\$ 10$ (Canadian) for their participation. All observers had normal or corrected-to-normal acuity when refracted for the test distance of $45 \mathrm{~cm}$.

Procedure. The stimuli were identical to those used in the conjunction search condition of Experiments 1A and 1B. All participants completed 144 trials, separated into four blocks of 36 trials each. However, observers in this experiment were instructed to maintain central fixation while searching for the target. Trials were discarded if the Eyegaze Development System indicated that an observer's eyes deviated from fixation by more than $1 \mathrm{~cm}\left(1.27^{\circ}\right)$ prior to response.

\section{Results}

Accuracy. A large eccentricity effect on accuracy was evident $[F(2,10)=34.93, p<.001]$. As Figure 6 indicates, there was a $40 \%$ reduction in accuracy between $3.82^{\circ}$ and $13.94^{\circ}$. At $13.94^{\circ}$, accuracy averaged $56 \%$, which was not significantly different from chance. In addition, performance was significantly less accurate on target-present $(78.6 \%)$ than on target-absent trials $[91.9 \%, F(1,5)=$ $14.89, p=.012]$.

Reaction times. Similar results were obtained with the RT data (see Figure 6). Between $3.82^{\circ}$ and $13.94^{\circ}$ RTs increased by $5 \mathrm{sec}$, yielding a significant eccentricity effect $[F(2,10)=14.06, p=.001]$. Observers were also slowed significantly on target-absent $(10.8 \mathrm{sec})$ versus target-present trials [3.9 sec, $F(1,5)=23.08, p=.005$ ]. However, in light of the high error rates, interpretation of the RT data is difficult.

Comparisons between Experiments $1 B$ and 2. Overall accuracy was marginally worse in Experiment 2 than in Experiment $1 \mathrm{~B}[F(1,10)=3.79, p=.080]$. The eccentricity effect was significant $[F(2,20)=31.84, p<.001]$, and an experiment $X$ eccentricity effect was also obtained $[F(2,20)=14.06, p<.001]$. As shown in Figure 6 , the greatest difference between the two experiments was found for the most eccentric targets. An experiment (2) $\times$ presence (2) ANOVA revealed a significant experiment effect $[F(1,10)=7.89, p=.018]$ and presence effect $[F(1,10)=30.77, p<.001]$, but no experiment $X$ presence interaction $(p=.631)$.

We also compared RT data from the two experiments. As Figure 6 depicts, RT differences across the experiments were largest for the most eccentric targets and on target-absent trials. An experiment (2) $\times$ eccentricity (3) ANOVA revealed a main effect of eccentricity $[F(2,20)=$ $18.32, p<.001]$ and an experiment $\times$ eccentricity interaction $[F(2,20)=9.82, p=.001]$. The experiment (2) $\times$ presence (2) ANOVA revealed a significant effect of experiment $[F(1,10)=8.84, p=.014]$ and presence $[F(1,10)=34.79, p<.001]$, along with an experiment $\times$ presence interaction $[F(1,10)=10.80, p=.008]$.

\section{Discussion}

The results of Experiment 2 buttress the argument that saccades are functional when unpracticed observers search for conjunction targets. Furthermore, the experiment $X$ eccentricity interactions obtained are consistent with the view that saccades, like RT, reflect differences in the attentional demands of the tasks.
Nakayama and Mackeben (1989, Experiment 1) reported that observers could identify conjunction targets in the absence of eye movements. However, their data are not at odds with our own. Their most eccentric target appeared at $5^{\circ}$, and our data suggest that saccades are functional only for greater eccentricities. They also indicated that participants performed at least 3,000 trials before data were collected. This leaves open the possibility that eye movements are needed early in practice, but that observers are able to process the displays without eye movements if they have sufficient exposure to the task (see S. B. Steinman, 1987). We explored this possibility in Experiment 3.

\section{EXPERIMENT 3}

The previous experiments showed that observers had difficulty processing conjunction search displays, but there is evidence of null display size effects in some conjunction search conditions. This occurs when conjunction targets are highly discriminable along one or more dimensions (McLeod et al., 1988; Nakayama \& Silverman, 1986; Wolfe et al., 1989) and when observers are given sufficient practice (S. B. Steinman, 1987). It is possible that these are the conditions that allow observers to search in groups large enough to encompass the entire display (Pashler, 1987; Poisson \& Wilkinson, 1992; Treisman \& Gormican, 1988; Zohary \& Hochstein, 1989). On the other hand, greater search efficiency might be attained by increasing the relative activation of target features (Cave \& Wolfe, 1989; Treisman \& Sato, 1990), perhaps omitting entire classes of items from inspection (Friedman-Hill $\&$ Wolfe, 1995). These are not mutually exclusive possibilities. If the relative activation hypothesis is correct, attention may be directed consistently away from distractors possessing nontarget features or toward distractors sharing particular feature values with the target. When feature selectivity is operating efficiently, the conjunction search task is translated to a feature search task. In this case, group size may expand to include all of the selected items in the display. To evaluate the possibilities, the two authors were given extensive practice at conjunction search. It was expected that saccadic involvement would diminish with experience and that eccentricity effects would be minimized.

\section{Method}

C.S. and K.J. were participants in the study. They were refracted at the test distance of $45 \mathrm{~cm}$ and wore corrections during all testing. The stimuli were identical to those used in the conjunction search condition of the previous experiments. Both observers completed 864 trials, separated into 12 testing sessions of 72 trials each.

\section{Results}

Figure 7 indicates that at initial testing both observers had long RTs and an elevated number of eye movements for the most eccentric targets. Average fixation durations and first fixation durations (not shown) tended to be relatively short, as would be expected given the large num- 

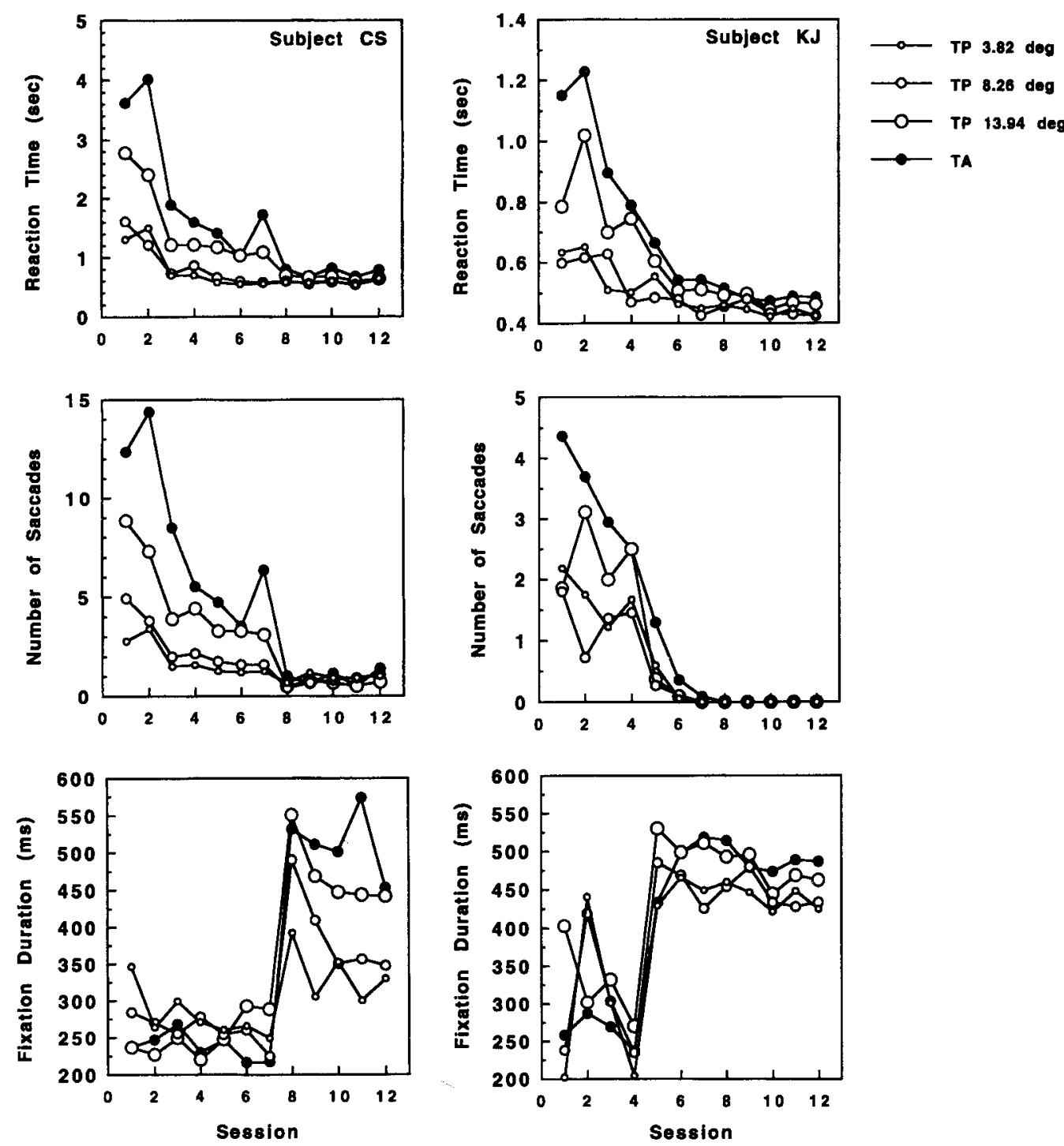

Figure 7. Experiment 3. Conjunction search reaction times (top panel), number of saccades (middle panel), and fixation durations (bottom panel) as a function of eccentricity, target presence, and practice session for C.S. (left panel) and K.J. (right panel).

ber of saccades. With practice, both observers improved to the point where eye movements were no longer made and RT became much less dependent on eccentricity. In addition, the difference between target-present and target-absent trials was minimized. The changes in RT were not due to a speed-accuracy tradeoff. Accuracy for C.S., shown in Table 2, was unrelated to practice. For K.J., there was improvement in accuracy with practice, particularly for more eccentric targets.

The functional relation between fixation duration and oculomotor involvement found in Experiments $1 \mathrm{~A}$ and $1 \mathrm{~B}$ was also obtained here. For C.S., power functions accounted for at least $70 \%$ of the variance in fixation durations through Session 7, beyond which saccades were made too infrequently for reliable curve fitting. For K.J., through Session 4 power functions accounted for at least $67 \%$ of the fixation duration variance. It is interesting to note that in both observers, the point in practice associated with a large decline in eye movements was also the point at which fixation durations showed a dramatic increase. This, too, is consistent with the view that the number of saccades indexes the amount of information that is being processed per fixation.

Even though the eccentricity effect was reduced with practice, it was not eliminated entirely. For C.S. in Session 12 , fixation durations averaged $330 \mathrm{msec}$ for $3.82^{\circ}$ targets but $441 \mathrm{msec}$ for targets at $13.94^{\circ}$. A similar effect was found for RTs. For K.J., this difference was $40 \mathrm{msec}$, like the difference in RTs. Though unlikely, it is possible that observers searched very large groups in a center-to- 
Table 2

Mean Accuracy and Standard Deviations for Experiment 3 as a Function of Session, Presence, and Eccentricity

\begin{tabular}{|c|c|c|c|c|c|c|c|c|}
\hline \multirow[b]{4}{*}{ Session } & \multicolumn{6}{|c|}{ TP } & & \\
\hline & \multicolumn{6}{|c|}{ Eccentricity } & & \\
\hline & \multicolumn{2}{|c|}{$3.82^{\circ}$} & \multicolumn{2}{|c|}{$8.26^{\circ}$} & \multicolumn{2}{|c|}{$133.94^{\circ}$} & \multicolumn{2}{|c|}{ TA } \\
\hline & $M$ & $S D$ & $M$ & $S D$ & $M$ & $S D$ & $M$ & $S D$ \\
\hline \multicolumn{9}{|c|}{ C.S. } \\
\hline 1 & 1 & 0 & 1 & 0 & .708 & .095 & .972 & .027 \\
\hline 2 & .917 & .083 & 1 & 0 & .833 & .112 & .944 & .055 \\
\hline 3 & 1 & 0 & 1 & 0 & .917 & .083 & 1 & 0 \\
\hline 4 & 1 & 0 & 1 & 0 & .583 & .149 & 1 & 0 \\
\hline 5 & 1 & 0 & 1 & 0 & .667 & .142 & .972 & .027 \\
\hline 6 & .917 & .083 & 1 & 0 & .583 & .149 & 1 & 0 \\
\hline 7 & 1 & 0 & 1 & 0 & .833 & .112 & 1 & 0 \\
\hline 8 & 1 & 0 & 1 & 0 & .833 & .112 & .972 & .027 \\
\hline 9 & 1 & 0 & 1 & 0 & .667 & .142 & .972 & .027 \\
\hline 10 & 1 & 0 & 1 & 0 & .917 & .083 & 1 & 0 \\
\hline 11 & 1 & 0 & 1 & 0 & .917 & .083 & 1 & 0 \\
\hline 12 & .917 & .083 & 1 & 0 & .583 & .149 & 1 & 0 \\
\hline \multicolumn{9}{|c|}{ K.J. } \\
\hline 1 & .917 & .083 & .833 & .112 & .583 & .149 & 1 & 0 \\
\hline 2 & 1 & 0 & .917 & .083 & .750 & .131 & 1 & 0 \\
\hline 3 & .750 & .131 & .917 & .083 & .667 & .042 & .972 & .027 \\
\hline 4 & 1 & 0 & .917 & .083 & .917 & .083 & .972 & .027 \\
\hline 5 & .917 & .083 & .917 & .083 & .667 & .142 & 1 & 0 \\
\hline 6 & 1 & 0 & .875 & .069 & .708 & .095 & .972 & .027 \\
\hline 7 & 1 & 0 & 1 & 0 & .833 & .112 & .944 & .055 \\
\hline 8 & .833 & .112 & .917 & .083 & .833 & .112 & .944 & .055 \\
\hline 9 & .917 & .083 & 1 & 0 & .833 & .112 & .944 & .055 \\
\hline 10 & 1 & 0 & 1 & 0 & .917 & .083 & .972 & .027 \\
\hline 11 & 1 & 0 & .917 & .083 & .750 & .131 & 1 & 0 \\
\hline 12 & .917 & .083 & 1 & 0 & .917 & .083 & 1 & 0 \\
\hline
\end{tabular}

Note-TP, target present; TA, target absent.

edge manner and that the differences in fixation durations reflect the movement of attention. It is also possible that these residual differences represent declines in resolution across the visual field.

\section{Discussion}

As observers gained experience with the task, their RT and saccade data became largely independent of eccentricity, and the mean number of saccades was reduced to less than one. Several other investigations have shown that conjunction search becomes independent of load with practice (e.g., S. B. Steinman, 1987), and this development of automaticity was revealed in both the eye movements and RTs of Experiment 3.

The remaining issue is one of mechanism. Examination of the scan paths for both K.J. and C.S. early in practice (see Figure 8 for examples) revealed a 3:1 bias for fixations to fall on or near white, not black, items. The selectivity seen in the fixation data, and reported previously in color $\times$ shape conjunction search RTs (Kim \& Cave, 1995 ) suggests that one mechanism underlying this change is the relative activation (Cave \& Wolfe, 1989; FriedmanHill \& Wolfe, 1995; Treisman \& Sato, 1990) of elements on the basis of their features. In the 2-D conjunction search task, selection on the basis of one feature value transforms the task into feature search. This transformation may be gradual. Early in practice, activation may be effective only on some proportion of trials, with the result that both eye movements and RT will be dependent on eccentricity. Over time, there will be an increase in the proportion of trials involving efficient selection. As such, one would expect the eccentricity effect to be eliminated.
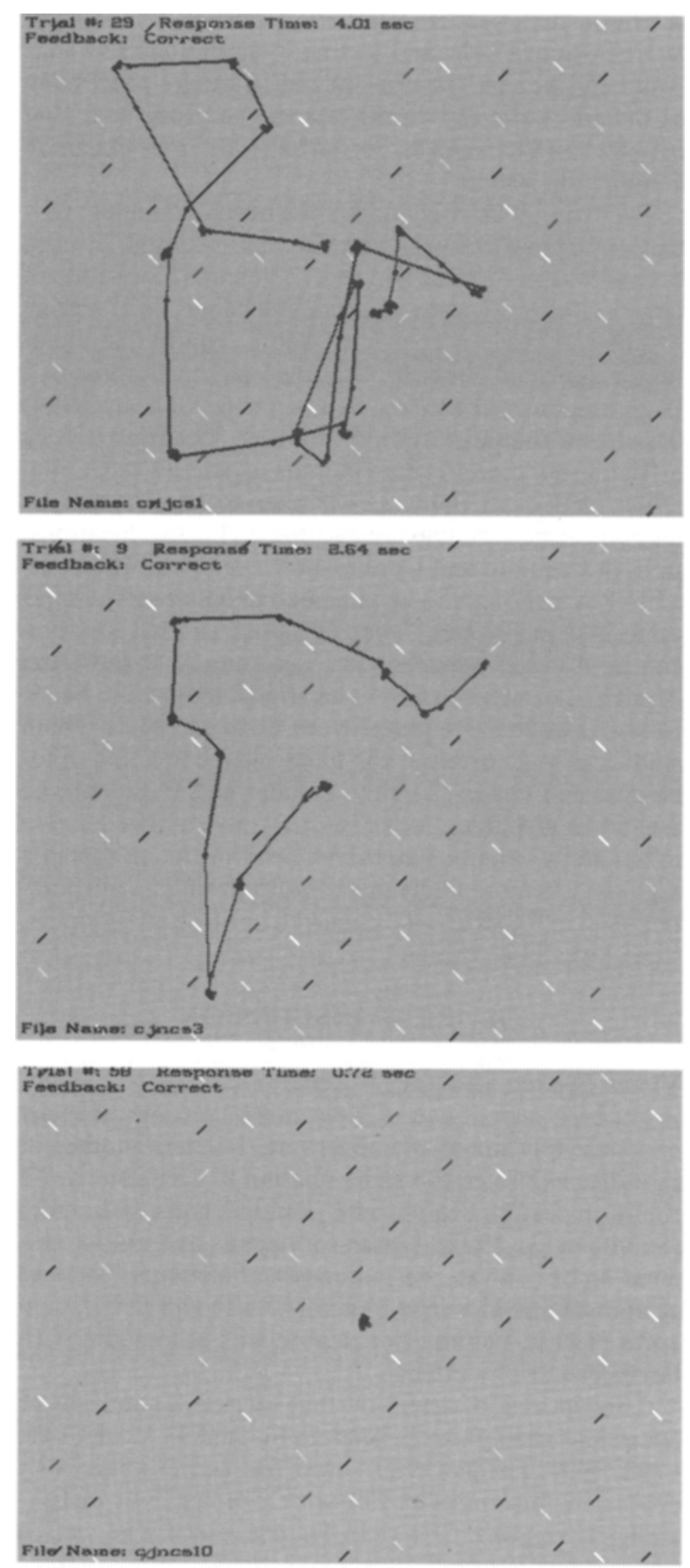

Figure 8. Experiment 3. Sample scan paths from target-present trials. Top panel: 1 st practice session. Middle panel: 3 rd practice session. Bottom panel: 10th practice session. 
As shown in Figure 8, early in practice the saccades describe a roughly circular scan path, an efficient means of searching the display. Later in practice, the circular shape of the scan path was maintained, but the diameter of the path was reduced until no eye movements were made. It is possible that experience allows observers to search displays in larger groups (Pashler, 1987; Poisson \& Wilkinson, 1992; Treisman \& Gormican, 1988; Zohary $\&$ Hochstein, 1989), and so smaller amplitude saccades could cluster near fixation and still allow for processing of the entire display. In this sense, the group scanning hypothesis (Treisman \& Gormican, 1988) may extend to conjunction search.

There might be concern that the development of automaticity apparent in the practice data resulted, in part, because observers learned the 12 potential locations of targets. Under this view, the near independence of performance and target eccentricity would require that attention is allocated either to 12 individual locations or to 2 larger regions centered on the diagonals. Duncan (1984) has shown that allocation to multiple locations is very difficult, but more recent findings (Castiello \& Umiltà, 1992; Kramer \& Hahn, 1995) seem to suggest that attention can be simultaneously allocated to two locations. In both Castiello and Umiltà and Kramer and Hahn, attention was thought to be allocated to only two small regions in opposite hemifields. It is not clear that attention can be divided between two locations with the same hemifield or more than two locations in opposite hemifields, as would be required in our study. In addition, both studies used an external cue to define the location of tobe-attended targets. Finally, Kramer and Hahn showed that when displays were onset suddenly, as was the case in our study, attention could not be allocated to separate regions. It is more likely that the improvement with practice seen in Experiment 3 occurred because attention became broadly distributed.

\section{GENERAL DISCUSSION}

\section{Visual Search and Target Eccentricity}

For both experienced (Experiment $1 \mathrm{~A}$ ) and inexperienced (Experiment 1B) observers, RT and number of saccades exhibited the same relation to eccentricity. As found in feature search with homogeneous distractors (Scialfa et al., 1994), search for objects of a unique orientation or contrast was independent of target location. In contrast, as in Carrasco et al. (1995) and Scialfa and Joffe (1997), conjunction search was associated with large eccentricity effects.

Conceptions of attention that rely on a spotlight or zoom lens metaphor (Eriksen \& St. James, 1986; Juola et al., 1991; Posner, 1980) allow for the possibility that processing resources may be widely or narrowly distributed across space. Generally, feature search is viewed as the result of a broad attentional distribution, whereas conjunction search is thought to require a more narrow focus. If resources within the focus of attention are distributed in a gradient-like manner (Downing \& Pinker,
1985; Egly \& Homa, 1984; Eriksen \& Schultz, 1979; Rizzolatti et al., 1987), one would expect greater eccentricity effects in conjunction than in feature search. To this extent, the present data use eccentricity effects to show that conjunction search often requires a narrower attentional focus than feature search.

\section{Functional Relations Between Attentional and Oculomotor Mechanisms}

Several lines of evidence suggest that the attentional demands of search tasks are indexed by saccadic eye movements as well as manual RT and accuracy. In Experiments $1 \mathrm{~A}, 1 \mathrm{~B}$, and 3 , the conditions producing large effects on RT yielded parallel effects in the number of saccades executed. The data of Experiment 3 indicate that practice reduces the eccentricity effect on RT and number of saccades. The preference shown by both observers to fixate items containing target features is an indicator of attentional and oculomotor coupling. Importantly, the fixation duration analyses of Experiments $1 \mathrm{~A}$ and $1 \mathrm{~B}$ show that a decomposition of global RTs may reveal conditionspecific changes in the processing demands of search that are not seen in RTs or saccades alone (Jacobs, 1986; Latimer, 1990; Locher \& Nodine, 1978; Rayner \& Fisher, 1987; Saitoh \& Okazaki, 1990; Scialfa \& Joffe, 1997).

\section{Conjunction Search}

Experiments $1 \mathrm{~A}$ and $1 \mathrm{~B}$ demonstrated that conjunction displays could not be processed independently of eccentricity. The ratio of target-absent to target-present saccades was approximately $2: 1$, but the number of eye movements executed was fewer than would be expected if observers needed to make an eye movement to process each element in the displays. As suggested by others (Poisson \& Wilkinson, 1992; Zohary \& Hochstein, 1989), the data open the possibility that Treisman and Gormican's (1988) group scanning hypothesis can be profitably extended to at least some conjunction search conditions. Another possibility is that conjunction search is guided by object features, so that efficiency is greater than would be expected from a purely random, serial, self-terminating search (Cave \& Wolfe, 1989; Friedman-Hill \& Wolfe, 1995; Wolfe, 1994).

The results of Experiment 2 indicate that in unpracticed observers, the eye movements made in conjunction search are functional, in the sense of being necessary, and are not a mere by-product of the greater time taken to identify a conjunction search target. When eye movements were precluded, accuracy decreased and RTs increased for more eccentric targets. In fact, interpretation of the RT data is problematic because observers were operating at chance levels in the most difficult condition.

Carrasco et al. (1995) hypothesized that the eccentricity effect found in conjunction search reflects diminished spatial resolution or increased lateral inhibition in noncentral vision. Taken alone, the results of Experiment 2 would be consistent with either hypothesis. However, increased peripheral lateral inhibition seems an unlikely explanation of the present data. If objects imaged on the 
peripheral retina were subject to more lateral inhibition, this interference would not diminish with experience, as seen in Experiment 3. On the other hand, "poorer resolution" may mean that peripheral localization is less precise. This could explain why only conjunction search is affected: Localization is unimportant in feature search. The practice data of Experiment 3 could be explained readily: In transforming the task to one of feature search, the observers minimized the importance of localization.

We did not find strong support for the rechecking hypothesis, but this conclusion must be qualified for at least two reasons. Rechecking did tend to occur more frequently on target-absent trials in Experiment 1B, a finding that is consistent with the focused rechecking hypothesis (Krueger, 1984). This effect was only marginally significant, but might have reached significance in a larger sample. Second, as noted, to the extent that group size includes more than one element, our analysis is based on systematically low estimates of rechecking. This, too, may have reduced effect sizes in the tests conducted. These caveats make it clear that without an independent estimate of group size, the rechecking hypothesis may be difficult to evaluate.

In summary, these data indicate that eye movements are not needed in feature search but are functional in conjunction search, and therefore provide an index of the differing attentional demands of these tasks. The saccade data yield results that parallel those found for RT and accuracy and therefore can provide convergent validity for models of attention and search. The featural selectivity seen in the saccade data provides additional information about the manner in which processing resources are guided in visual search and suggests one mechanism that can allow for efficient conjunction search. Finally, we have shown that there is a functional relation between saccades and fixation durations that deserves further study.

\section{REFERENCES}

Binello, A., Mannan, S., \& Ruddock, K. H. (1995). The characteristics of eye movements made during visual search with multi-element stimuli. Spatial Vision, 9, 343-362.

Braun, D., \& Breitmeyer, B. G. (1988). Relationship between directed visual attention and saccadic reaction times. Experimental Brain Research, 73, 546-552.

Carrasco, M., Evert, D. L., Chang, I., \& Katz, S. M. (1995). The eccentricity effect: Target eccentricity affects performance on conjunction searches. Perception \& Psychophysics, 57, 1241-1261.

Castiello, U., \& Umiltà, C. (1992). Splitting focal attention. Journal of Experimental Psychology: Human Perception \& Performance, 18, 837-848.

CAVE, K. R., \& WolfE, J. M. (1989). Modeling the role of parallel processing in visual search. Cognitive Psychology, 22, 225-271.

Cleveland, D., \& Cleveland, N. (1992, January). Eyegaze eyetracking system. Paper presented at the Eleventh Monte Carlo International Forum on New Images, Monte Carlo.

Deubel, H., \& Schneider, W. X. (1996). Saccade target selection and object recognition: Evidence for a common attentional mechanism. Vision Research, 36, 1827-1837.

Downing, C. J., \& Pinker, S. (1985). The spatial structure of visual attention. In M. I. Posner \& O. S. M. Martin (Eds.), Attention and performance $X I$ (pp. 475-492). Hillsdale, NJ: Erlbaum.

DUNCAN, J. (1984). Selective attention and the organization of visual information. Journal of Experimental Psychology: General, 113, 501-517.
DunCAN, J., \& HumphreYs, G. W. (1989). Visual search and stimulus similarity. Psychological Review, 96, 433-458.

EGLY, R., \& HoMA, D. (1984). Sensitization of the visual field. Journal of Experimental Psychology, 10, 778-793.

ERIKSEN, C. W., \& SCHULTZ, D. W. (1979). Information processing and visual search: A continuous flow conception and experimental results. Perception \& Psychophysics, 25, 249-263.

ERIKSEN, C. W., \& ST. JAMES, J. D. (1986). Visual attention within and around the field of focal attention: A zoom lens model. Perception \& Psychophysics, 40, 225-240.

Findtay, J. M., \& Ka POUla, Z. (1992). Scrutinization, spatial attention, and the spatial programming of saccadic eye movements. Quarterly Journal of Experimental Psychology, 45A, 633-647.

Friedman-Hill, S., \& WolfE, J. M. (1995). Second-order parallel processing: Visual search for the odd item in a subset. Journal of Experimental Psychology: Human Perception \& Performance, 21, 531551.

Goldberg, M. E., \& Bruce, C. J. (1985). Cerebral cortical activity associated with the orientation of visual attention in the rhesus monkey. Vision Research, 25, 471-481.

Goldberg, M. E., Bushnell, M. C., \& Bruce, C. J. (1986). The effect of attentive fixation on eye movements evoked by electrical stimulation of the frontal eye fields. Experimental Brain Research, 61, 579-584.

GoldBerg, M. E., \& WurTZ, R. H. (1972). Activity of superior colliculus in behaving monkey. II. Effect of attention on neuronal responses. Journal of Neurophysiology, 35, 560-574.

HeNDERSON, J. M. (1993). Visual attention and saccadic eye movements. In R. Groner, G. d'Ydewalle, \& R. Parham (Eds.), From eye to mind: Information acquisition in perception, search and reading (pp. 37-50). Amsterdam: Elsevier.

Henderson, J. M., \& Ferreira, F. (1990). Effects of foveal processing difficulty on the perceptual span in reading: Implications for attention and eye movement control. Journal of Experimental Psychology: Learning, Memory, \& Cognition, 16, 417-429.

Henderson, J. M., Pollatsek, A., \& Rayner, K. (1989). Covert visual attention and extrafoveal information use during object identification. Perception \& Psychophysics, 45, 196-208.

HoFfMaN, J. E. (in press). Visual attention and eye movements. In H. Pashler (Ed.), Attention. London: University College London Press.

Hoffman, J, E., \& Subramaniam, B. (1995). The role of visual attention in saccadic eye movements. Perception \& Psychophysics, 57, 787-795.

JACOBS, A. M. (1986). Eye-movement control in visual search: How direct is visual span control? Perception \& Psychophysics, 39, 47-58.

Juola, J. F., Bouwhuis, D. G., Cooper, E. E., \& WARNER, C. B. (1991). Control of attention around the fovea. Journal of Experimental Psychology: Human Perception \& Performance, 17, 125-141.

Kaptein, N. A., Theeuwes, J., \& van der Heijden, A. H. C. (1995). Search for a conjunctively defined target can be selectively limited to a color-defined subset of elements. Journal of Experimental Psychology: Human Perception \& Performance, 21, 1053-1069.

KHURANA, B., \& KowLER, E. (1987). Shared attentional control of smooth eye movement and perception. Vision Research, 27, 1603-1618.

KIM, M.-S., \& CAVE, K. R. (1995). Spatial attention in visual search for features and feature conjunctions. Psychological Science, 6, 376-380.

KLEIN, R., \& FARrell, M. (1989). Search performance without eye movements. Perception \& Psychophysics, 46, 476-482.

Kowler, E., Anderson, E., Dosher, B., \& Blaser, E. (1995). The role of attention in the programming of saccades. Vision Research, 35, 1897-1916.

Kramer, A. F., \& HahN, S. (1995). Splitting the beam: Distribution of attention over noncontiguous regions of the visual field. Psychological Science, 6, 381-386.

KRUEGER, L. E. (1984). Self-termination in same-different judgments: Multiletter comparison with simultaneous and sequential presentation. Journal of Experimental Psychology: Learning, Memory, \& Cognition, 10, 271-284.

LATIMER, C. R. (1990). Eye-movement indices of form perception: Some methods and preliminary results. In R. Groner, G. d'Ydewalle, \& R. Parham (Eds.), From eye to mind: Information acquisition in perception, search and reading (pp. 41-57). Amsterdam: Elsevier.

LOCHER, P. J., \& NODINE, C. F. (1978). Influence of stimulus symmetry on visual scanning patterns. Perception \& Psychophysics, 13, 408-412. 
Lynch, J. C., Mountcastle, V. B., Talbot, W. H., \& Yin, T. C. T. (1977). Parietal lobe mechanisms for directed visual attention. Journal of Neurophysiology, 40, 362-389.

McConkie, G. W., \& Rayner, K. (1975). The span of the effective stimulus during a fixation in reading. Perception \& Psychophysics, 17, 578-586.

MCLeod, P., Driver, J., \& Crisp, J. (1988). Visual search for a conjunction of movement and form is parallel. Nature, 332, 154-155.

Miura, T. (1990). Active function of eye movement and useful field of view in a realistic setting. In R. Groner, G. d'Ydewalle, \& R. Parham (Eds.), From eye to mind: Information acquisition in perception, search and reading (pp. 119-127). Amsterdam: Elsevier.

MorRison, R. E. (1984). Manipulation of onset in reading: Evidence for parallel programming of saccades. Journal of Experimental Psychology: Human Perception \& Performance, 10, 667-682.

MUNOZ, D. P., \& WURTZ, R. H. (1992). Role of the rostral superior colliculus in active visual fixation and execution of express saccades. Journal of Neurophysiology, 67, 1000-1002.

Nakayama, K., \& MackebEN, M. (1989). Sustained and transient components of focal visual attention. Vision Research, 29, 1625-1641.

Nakayama, K., \& Silverman, G. H. (1986). Serial and parallel processing of visual feature conjunctions. Nature, 320, 264-265.

NatTKemper, D., \& Prinz, W. (1986). Saccade amplitude determines fixation duration: Evidence from continuous search. In J. K. O'Regan \& A. Lévy-Schoen (Eds.), Eye movements: From physiology to cognition (pp. 285-292). Amsterdam: North-Holland.

NothDurf, H. C. (1985). Orientation sensitivity and texture segmentation in patterns with different line orientation. Vision Research, 25 , 551-560.

PASHLER, H. (1987). Target-distractor discriminability in visual search. Perception \& Psychophysics, 41, 285-292.

Pierrot-Diseilligny, C., Rivaud, S., Penet, C., \& Rigolet, M.-H. (1987). Latencies of visually guided saccades in unilateral hemispheric cerebral lesions. Annals of Neurology, 21, 138-148.

Plude, D. J., \& Doussard-Roosevelt, J. A. (1989). Aging, selective attention, and feature integration. Psychology \& Aging, 4, 98-115.

PoIsson, M. E., \& WILKInSON, F. (1992). Distractor ratio and grouping processes in visual conjunction search. Perception, 21, 21-38.

POSNER, M. I. (1980). Orienting of attention. Quarterly Journal of Experimental Psychology, 32, 3-25.

Posner, M. I., SNyder, C. R. R., \& Davidson, B. J. (1980). Attention and the detection of signals. Journal of Experimental Psychology: General, 109, 160-174.

RAYNER, K., \& FiSHER, D. L. (1987). Letter processing during eye fixations in visual search. Perception \& Psychophysics, 42, 87-100.

Rizzolatti, G., Riggio, L., Dascola, 1., \& Umiltà, C. (1987). Reorienting attention across the horizontal and vertical meridians: Evidence in favor of a premotor theory of attention. Neuropsychologia, 25, 31-40.

SAITOH, O., \& OKAZAKI, Y. (1990). Eye movements: A tool of chronometry in visual information processing. In R. Groner, G. d'Ydewalle, $\&$ R. Parham (Eds.), From eye to mind: Information acquisition in perception, search and reading (pp. 23-40). Amsterdam: Elsevier.

SCHILlER, P. H., \& SANDELL, J. H. (1983). Interactions between visually and electrically elicited saccades before and after superior colliculus and frontal eye field ablations in the rhesus monkey. Experimental Brain Research, 49, 381-392.

SCHNEIDER, W. (1985). Toward a model of attention and the development of automatic processing. In M. I. Posner and O. S. M. Martin (Eds.), Attention \& Performance XI (pp. 475-492). Hillsdale, NJ: Erlbaum.

SCHneider, W., Pimm-Smith, M., \& Worden, M. (1994). Neurobiology of attention and automaticity. Current Opinion in Neurobiology, $\mathbf{4}$, 177-182.

SCHNeIder, W., \& ShifFrin, R. M. (1977). Controlled and automatic human information processing: I. Detection, search, and attention. Psychological Review, 84, 1-66.

Scialfa, C. T., \& JofFe, K. M. (1997). Age differences in feature and conjunction search: Implications for theories of visual search and generalized slowing. Aging, Neuropsychology, \& Cognition, 4, 227-246.

SCIALFA, C. T., \& KLINE, D. W. (1988). Effects of noise type and retinal eccentricity on age differences in identification and localization. Journal of Gerontology: Psychological Sciences, 43, P91-99.

Scialfa, C. T., Kline, D. W., \& Lyman, B. J. (1987). Age differences in target identification as a function of retinal location and noise level: Examination of the useful field of view. Psychology \& Aging, 2, 14-19. Scialfa, C. T., Thomas, D. M., \& JofFe, K. M. (1994). Age differences in the useful field of view: An eye movement analysis. Optometry \& Vision Science, 71, 1-7.

Sheppard, M., Findlay, J. M., \& Hockey, R. J. (1986). The relationship between eye movements and spatial attention. Quarterly Journal of Experimental Psychology, 38A, 475-491.

Shibutani, H., Sakata, H., \& Hyvarinen, J. (1984). Saccade and blinking evoked by microstimulation of the posterior parietal association cortex of the monkey. Experimental Brain Research, 55, 1-8.

SPARKS, D. L., \& MAYS, L. E. (1983). Spatial localization of saccade targets. I. Compensation for stimulation-induced perturbations in eye position. Journal of Neurophysiology, 49, 45-63.

SPERLING, G., \& ReEves, A. (1986). Attentional gating in short-term visual memory. Psychological Review, 93, 180-206.

Steinman, R. M. (1986). Eye movement. Vision Research, 26, 1389-1400 Steinman, S. B. (1987). Serial and parallel search in pattern vision. Perception, 16, 389-398.

TheEuwes, J., \& Koo1, F. L. (1994). Parallel search for a conjunction of contrast polarity and shape. Vision Research, 34, 3013-3016.

Treisman, A. (1988). Features and objects: The fourteenth Bartlett memorial lectures. Quarterly Journal of Experimental Psychology, 40A, 210-237.

Treisman, A., \& GormiCAN, S. (1988). Feature analysis in early vision: Evidence from search asymmetries. Psychological Review, 95, 15-48.

Treisman, A., \& Sato, S. (1990). Conjunction search revisited. Journal of Experimental Psychology: Human Perception \& Performance, 16, 459-478.

Treisman, A., \& Souther, J. (1985). Search asymmetry: A diagnostic for preattentive processing of separable features. Journal of Experimental Psychology: General, 114, 285-310.

VIVIANI, P., \& SWENSSON, R. G. (1982). Saccadic eye movements to peripherally discriminated visual targets. Journal of Experimental Psychology: Human Perception \& Performance, 8, 113-126.

WOLFE, J. M. (1994). Guided Search 2.0: A revised model of visual search. Psychonomic Bulletin \& Review, 1, 202-238.

Wolfe, J. M., Cave, K. R., \& Franzel, S. L. (1989). Guided search: An alternative to the modified feature integration model for visual search. Journal of Experimental Psychology: Human Perception \& Performance, 15, 419-433.

YOUNG, L. R., \& SHEENA, D. (1975). Survey of eye movement recording methods. Behavior Research Methods \& Instrumentation, 7, 397-429.

ZACKS, J. L., \& ZACKS, R. T. (1993). Visual search times assessed without reaction times: A new method and an application to aging. Journal of Experimental Psychology: Human Perception \& Performance, 19, 798-813.

ZeLiNSKy, G. (1996). Using eye saccades to assess the selectivity of search movements. Vision Research, 36, 2177-2187.

ZOHARY, E., \& HoCHSTEIN, S. (1989). How serial is serial processing in vision? Perception, 18, 191-200.

\section{NOTES}

1. Given that first fixations were significantly longer than average fixations, we also determined power functions after removing the first fixation duration from the data. As expected, specific values of parameter estimates changed, but the trends remained. The intercepts averaged 217,183 , and $290 \mathrm{msec}$ for feature orientation, feature contrast, and conjunction search, respectively. The exponents averaged -.25 , -.26 , and -.27 for these same conditions and the corresponding $r^{2}$ were $.49, .77$, and .44 .

2. As in Experiment 1A, we determined power functions after removing the first fixation durations from the data and found similar trends. The intercepts averaged 262,245 , and $369 \mathrm{msec}$ for feature orientation, feature contrast, and conjunction search, respectively. The exponents averaged $-.26,-.06$, and -.32 for these same conditions and the corresponding $r^{2}$ s were $.47, .36$, and .55 .

3. Similar results were obtained when first fixations were eliminated.

(Manuscript received January 12, 1996;

revision accepted for publication August 18, 1997.) 